\section{Improvement of Arterial Stiffness in the} Transition From Acute Decompensated Heart Failure to Chronic Compensated Heart Failure
Address for correspondence: Sang Hong Baek, MD, Division of Cardiology, Department of Internal Medicine, Seoul St. Mary's Hospital, Banpo-Dong, Seocho-Gu,

Seoul, Korea,

whitesh@catholic.ac.kr

\author{
Dong-Bin Kim, MD; Sang Hong Baek, MD; Sung Won Jang, MD; Sung-Ho Her, MD; \\ Dong-II Shin, MD; Chan Seok Park, MD; Hoon Joon Park, MD; Pum Joon Kim, MD; \\ Hae Ok Jung, MD; Ki Bae Seung, MD \\ Division of Cardiology, Department of Internal Medicine, College of Medicine, The Catholic \\ University of Korea, Seoul, Republic of Korea
}

\begin{abstract}
Background: Pulse wave velocity (PWV) is a well-established marker for aortic stiffness and may be a prognostic factor in heart failure (HF). This study investigates whether PWV changes as patients transition from acute decompensated heart failure (ADHF) to chronic compensated heart failure (CCHF).

Hypothesis: Arterial stiffness is related with the development of HF.

Methods: Regional PWV was prospectively measured using noninvasive applanation tonometry in consecutive ADHF patients $(n=55)$. PWV measurements of 45 patients were taken at admission and 3-month follow-up (F/U).

Results: Central and upper-extremity PWV, but not lower-extremity PWVs, were found to have improved after 3 months compared with the admission PWV (central: $8.73 \pm 1.17$ vs $8.39 \pm 0.99 \mathrm{~m} / \mathrm{s}, P=0.018$; upper extremity: $8.59 \pm 0.84$ vs $8.33 \pm 0.82 \mathrm{~m} / \mathrm{s}, P=0.028$ ). Multivariate logistic regression analyses revealed that low-density lipoprotein cholesterol was significantly associated with the change of PWV in HF (odds ratio: $1.037,95 \%$ confidence interval: 1.003-1.071, $P=0.030$ ). In preserved left ventricular ejection fraction patients $(n=26)$ and ischemic patients $(n=31)$, central and upper-extremity PWVs improved over the admission PWV at 3-month $\mathrm{F} / \mathrm{U}$.

Conclusions: The present results indicate that central and upper-extremity PWVs, but not lower-extremity PWV, are increased in ADHF and improve as patients transition from ADHF to CCHF.
\end{abstract}

\section{Introduction}

Heart failure (HF) is a chronic illness affecting $1 \%-2 \%$ of the population, and it is characterized by episodes of decompensation often requiring hospitalization. ${ }^{1}$ A complex blend of structural and functional alterations accounts for the progressive nature of $\mathrm{HF}^{2}$ but the exact mechanisms underlying this disease remain poorly defined.

Vessel stiffness is known to be a factor in $\mathrm{HF}$ pathophysiology. ${ }^{3}$ Central aortic stiffness may accelerate HF development in vulnerable patients by increasing the systolic load and worsening ventricular-vascular coupling. Even a slight increase in the left ventricular (LV) afterload can cause major changes in hemodynamic and clinical profiles in HF patients. ${ }^{4}$

Pulse wave velocity (PWV) is currently measured in clinical settings and reflects arterial stiffness. Pulse wave velocity is increased in patients with diabetes mellitus (DM) and coronary artery disease ${ }^{5,6}$ and is considered a prognostic indicator for cardiovascular disease in healthy

The authors have no funding, financial relationships, or conflicts of interest to disclose. people and patients with hypertension, DM, or end-stage renal failure. ${ }^{7-11}$

To date, no studies have reported regional PWV in acute decompensated heart failure (ADHF) or change in regional $\mathrm{PWV}$ as patients undergo a transition from $\mathrm{ADHF}$ to chronic compensated heart failure (CCHF). The present study investigates the regional PWV in ADHF patients and also examines whether PWV changes as patients transition from $\mathrm{ADHF}$ to $\mathrm{CCHF}$.

\section{Methods \\ Patients}

Patient eligibility criteria for this prospective study were age $\geq 40$ years, New York Heart Association class III or IV, management in an HF program with optimized medical therapy for $\geq 3$ months before enrollment, and $\geq 1$ HF-related hospitalization or emergency-department visit requiring intravenous treatment prior to enrollment. The patient exclusion criteria were acute myocardial infarction (MI) within 3 months, peripheral arterial disease, arrhythmia, or renal failure where the creatinine level was $\geq 2.0 \mathrm{mg} / \mathrm{dL}$. Patients with ischemic heart disease were defined as those 
with a documented history of MI, typical angina, and angiographic evidence of coronary artery disease. Age, sex, and body mass index (BMI) were included as clinical variables, and the medical history of each subject, including medication use, was confirmed from a comprehensive review of the medical records. The blood pressure, heart rate, and PWV were simultaneously measured. The protocol of this study was reviewed and approved by the ethics committee of Seoul St. Mary's Hospital and conducted according to the Declaration of Helsinki.

\section{Pulse Wave Velocity Measurement}

PWV represents the rate at which a pulse wave is transmitted through the blood vessels and was calculated as pulse transit time (ie, the time taken for the wave to pass between 2 points). For PWV measurements, patients were placed in a supine position, allowed to stabilize for $\geq 5$ minutes, and then a noninvasive automatic pulse wave analyzer (PP-1000; Hanbyul Meditech, Jeonju, Korea) was used to measure PWV. The initial PWV for $\mathrm{ADHF}$ patients was determined within 48 hours of admission. Tonometers were placed at the location of the arterial pulse waves for the left carotid artery, radial artery, femoral artery, and dorsalis pedis artery. Electrocardiographic (ECG) sensors were attached bilaterally to the wrists using an electrode. A phonocardiography sensor, designed using a piezopolymer film contact microphone, was placed in the second intercostal space of the left border of the sternum to check for cardiac sound. PWV was calculated using an intersecting tangent algorithm, which detected the starting point of each pulse wave. The PWV values were calculated based on the extraction of foot-to-foot transit time, which was determined by the time difference between the upstroke points of 2 pulse waves obtained at 2 different sites. Six signals (ECG, phonocardiography, and 4 pulse waves) from the carotid, radial, femoral, and dorsalis pedis arteries on the left side of the body were recorded simultaneously for a duration of 10 seconds. Surface distances for the measurement of PWV were calculated from the subject's height and from anthropomorphic data from the Korean population. For the automatic determination of PWV values, the surface distance between the 2 recording sites of a pulse wave was put into the system to allow for the calculation of PWV values. Carotid-femoral artery PWV was considered central PWV, carotid-radial artery PWV was considered upper-extremity PWV, and femoral-dorsalis pedis artery PWV was considered lower-extremity PWV. All PWVs were recorded synchronously. The coefficients of variation were $3.6 \%, 4.5 \%$, and $3.9 \%$ for central PWV, upper-extremity PWV, and lower-extremity PWV, respectively.

\section{B-type Natriuretic Peptide and Echocardiography Measurement}

Blood samples were collected at hospital admission in ethylenediaminetetraacetic acid tubes for B-type natriuretic peptide measurement (Bayer Diagnostics, Tarrytown, NY). Peptide levels were measured using a chemiluminescence immunoassay method previously described. ${ }^{12}$
Echocardiography was performed within 48 hours of admission using commercially available ultrasound systems (Acuson Sequoia 512; Siemens Medical Solutions, Mountain View, CA; and Vivid 5; GE Medical Systems, Waukesha, WI). Left ventricular ejection fraction (LVEF) was measured using the quantitative 2-dimensional biplane volumetric modified Simpson method, using 4- and 2-chamber views. Left ventricular systolic dysfunction was defined as an LVEF $<40 \%$.

Diastolic function was assessed using pulse-wave Doppler measurements at the tip of the mitral valve and pulmonary vein, and tissue Doppler measurements at the septal mitral annulus. The early-stage maximal diastolic velocity $\mathrm{E}$ wave ( $\mathrm{E}$ velocity) and the late-stage atrial systolic rate of the blood flow A wave (A velocity) were measured. The movement velocity of the mitral annulus was measured using an apical 4-chamber view and a tissue Doppler imaging study. The early-stage diastolic mitral annulus maximal velocity ( $e^{\prime}$ velocity) was obtained. The ratio of maximal early transmitral velocity to early diastolic tissue velocity $\left(E / \mathrm{e}^{\prime}\right)$ was also acquired for the evaluation of diastolic dysfunction associated with LV filling pressure. Digital echocardiographic data containing a minimum of 3 consecutive measurements were acquired, transferred to a server for storage, and interpreted by 2 independent cardiologists, who were blinded from each other's readings. We used the means of the 2 interpretation values.

\section{Follow-Up Observation}

Blood pressure and regional PWV were determined 3 months after the date of ADHF patient admission. Followup $(\mathrm{F} / \mathrm{U})$ PWVs were obtained using the same methods as described above. Medications administered at the outpatient clinic were recorded in $\mathrm{F} / \mathrm{U}$ studies. Patients in whom $\mathrm{ADHF}$ recurred during the $\mathrm{F} / \mathrm{U}$ period were excluded from the study. We also investigated clinical outcomes (cardiac death and readmission for $\mathrm{ADHF}$ ) at 6 months after the date of $\mathrm{ADHF}$ patient admission.

\section{Statistical Analysis}

Results are presented as mean \pm standard deviation (SD) for continuous variables, and as frequency percentage for categorical variables. Intergroup comparisons were made using independent Student $t$ tests and $\chi^{2}$ tests. Further, we analyzed change in PWV between $\mathrm{ADHF}$ and $\mathrm{CCHF}$ using paired $t$ tests. Multivariate logistic regression analyses were used to identify the predictors of the change in PWV, and univariate variables with $P<0.20$ were entered into the multivariate logistic models. We analyzed changes of medication in the ADHF-to-CCHF transition using a $\chi^{2}$ test. A $P$ value $<0.05$ was considered to indicate statistical significance.

\section{Results}

The study enrolled 55 consecutive ADHF patients. There was no difference in baseline characteristics between the systolic HF and diastolic HF groups, except for a higher proportion of patients taking digoxin in the systolic HF group. There was a trend toward an increased prevalence 
of hypertension in the diastolic HF group; however, likely due to the small number of patients, this failed to reach statistical significance $(P=0.099)$ (Table 1$)$.

$\mathrm{F} / \mathrm{U}$ PWVs were measured in 45 of the 55 patients at 3 months (7 patients were readmitted for recurrent $\mathrm{ADHF}$, and thus excluded from the study; 3 were lost to outpatient clinic F/U). Central and upper-extremity PWVs improved at 3-month $\mathrm{F} / \mathrm{U}$ over the initial admission (central: $8.73 \pm 1.17$ vs $8.39 \pm 0.99 \mathrm{~m} / \mathrm{s}, P=0.018$; upper-extremity: $8.59 \pm 0.84$ vs $8.33 \pm 0.82 \mathrm{~m} / \mathrm{s}, P=0.028)$. In contrast, lowerextremity PWV did not change over time $(9.57 \pm 1.42$ vs $9.48 \pm 1.51 \mathrm{~m} / \mathrm{s}, P=0.749$ ) (Figure 1).

We used the following variables in simple logistic regression: age, male sex, BMI, DM, hypertension, smoking, history of MI, creatinine, total cholesterol, lowdensity lipoprotein cholesterol (LDL-C), high-sensitivity C-reactive protein, and LVEF. Simple logistic regression analysis showed that the $P$ values of BMI and LDL-C were 0.149 and 0.026 , respectively, but only LDL-C was significant in the multivariate logistic model (odds ratio: 1.037, 95\% confidence interval: 1.003-1.071, $P=0.030$ ).

We analyzed the patients divided into 2 groups, above and below the threshold LVEF of $40 \%$. In patients with LVEF $\leq 40 \%(\mathrm{n}=19)$, none of the F/U PWVs changed over time. In preserved LVEF patients $(n=26)$, central and upperextremity PWVs improved at 3-month F/U over the initial admission PWV (central: $8.87 \pm 1.20$ vs $8.50 \pm 1.05 \mathrm{~m} / \mathrm{s}$, $P=0.044$; upper extremity: $8.58 \pm 0.85$ vs $8.21 \pm 0.74 \mathrm{~m} / \mathrm{s}$, $P=0.026$ ). In contrast, the lower-extremity PWV did not change over time (Table 2). We also divided the group according to presence of ischemia; the $45 \mathrm{~F} / \mathrm{U}$ patients comprised 31 ischemic and 14 nonischemic subjects. Central PWV and upper-extremity PWV improved in the ischemic group (central: $8.82 \pm 1.10$ vs $8.44 \pm 0.95 \mathrm{~m} / \mathrm{s}, P=0.008$; upper extremity: $8.59 \pm 0.87$ vs $8.22 \pm 0.82 \mathrm{~m} / \mathrm{s}, P=0.011$ ), whereas lower-extremity PWV did not change over time in the ischemic group. In the nonischemic group, none of the F/U PWVs changed over time (Table 2). We performed clinical $\mathrm{F} / \mathrm{U}$ at 6 months. Due to loss of outpatient clinic $\mathrm{F} / \mathrm{U}$, we could not obtain the clinical information of 1 patient in the group where central and upper-extremity PWVs improved and 1 patient in the group where central and upper-extremity PWVs did not improve. Six clinical events occurred in the group where central and upper-extremity PWVs improved $(\mathrm{n}=25)$ and 4 in the group where central and upper-extremity PWVs did not improve $(\mathrm{n}=18)$. There was no statistical difference in incidence of clinical outcomes between the 2 groups $(P=0.914)$.

Hemodynamic parameters in PWV measurement and medication use in the $45 \mathrm{~F} / \mathrm{U}$ patients, before admission and at the 3-month $\mathrm{F} / \mathrm{U}$, were found to be similar (Table 3).

\section{Discussion}

This is the first study to show that central and upperextremity PWVs were increased in ADHF compared with CCHF. This finding supports that one of the mechanisms in developing ADHF is increased arterial stiffness. ${ }^{13}$

$\mathrm{ADHF}$ is a collection of clinical syndromes with varying and poorly understood pathophysiological mechanisms. There are several mechanisms to explain this phenomenon;
Table 1. Baseline Characteristics of ADHF

\begin{tabular}{|c|c|c|c|c|}
\hline & $\begin{array}{l}\text { ADHF, } \\
n=55\end{array}$ & $\begin{array}{l}\text { Systolic HF, } \\
n=24\end{array}$ & $\begin{array}{c}\text { Diastolic HF, } \\
\qquad n=31\end{array}$ & $P$ Value $^{\mathrm{a}}$ \\
\hline Age, $y$ & $65.4 \pm 12.6$ & $64.4 \pm 12.8$ & $66.2 \pm 12.6$ & 0.593 \\
\hline Male & $25(46)$ & $10(42)$ & $15(48)$ & 0.785 \\
\hline BMI, $\mathrm{kg} / \mathrm{m}^{2}$ & $22.86 \pm 2.94$ & $23.02 \pm 3.03$ & $22.74 \pm 2.90$ & 0.720 \\
\hline DM & $15(27)$ & $7(29)$ & $8(26)$ & 1.000 \\
\hline Hypertension & $31(56)$ & $17(71)$ & $14(45)$ & 0.099 \\
\hline Smoking & $13(24)$ & $6(24)$ & $7(28)$ & 1.000 \\
\hline History of prior MI & $41(75)$ & 19 (79) & $22(71)$ & 0.547 \\
\hline \multicolumn{5}{|l|}{ Current medication } \\
\hline$\beta$-Blocker & $35(64)$ & $13(54)$ & $22(71)$ & 0.262 \\
\hline CCB & $19(35)$ & $7(29)$ & $12(39)$ & 0.571 \\
\hline ACEI or ARB & $45(82)$ & $21(88)$ & $24(77)$ & 0.486 \\
\hline Digoxin & $23(42)$ & $15(63)$ & $8(21)$ & 0.001 \\
\hline Diuretic & $33(60)$ & $17(71)$ & $16(52)$ & 0.175 \\
\hline $\begin{array}{l}\text { Lipid-lowering } \\
\text { agent }\end{array}$ & $41(75)$ & $19(79)$ & $21(68)$ & 0.380 \\
\hline $\mathrm{Cr}, \mathrm{mg} / \mathrm{dL}$ & $0.99 \pm 0.29$ & $1.03 \pm 0.30$ & $0.96 \pm 0.29$ & 0.364 \\
\hline $\mathrm{TC}, \mathrm{mg} / \mathrm{dL}$ & $172.8 \pm 53.4$ & $166.5 \pm 39.3$ & $177.7 \pm 62.4$ & 0.455 \\
\hline $\mathrm{LDL}-\mathrm{C}, \mathrm{mg} / \mathrm{dL}$ & $90.9 \pm 27.6$ & $88.6 \pm 27.6$ & $92.5 \pm 27.9$ & 0.631 \\
\hline BNP, pg/mL & $1039.6 \pm 934.2$ & $1092.3 \pm 966.7$ & $997.5 \pm 921.8$ & 0.715 \\
\hline $\mathrm{Hs}$-CRP, mg/dL & $2.31 \pm 3.44$ & $1.94 \pm 2.8$ & $2.59 \pm 3.89$ & 0.508 \\
\hline \multicolumn{5}{|c|}{ Echocardiographic findings } \\
\hline $\mathrm{EF}, \%$ & $39.30 \pm 8.03$ & $31.88 \pm 5.89$ & $45.05 \pm 3.353$ & $<0.001$ \\
\hline $\begin{array}{l}\text { Early mitral inflow, } \\
E(m / s)\end{array}$ & $0.75 \pm 0.23$ & $0.74 \pm 0.21$ & $0.76 \pm 0.25$ & 0.889 \\
\hline $\begin{array}{l}\text { Late mitral inflow, } \\
A(\mathrm{~m} / \mathrm{s})\end{array}$ & $0.66 \pm 0.29$ & $0.58 \pm 0.321$ & $0.72 \pm 0.25$ & 0.150 \\
\hline E/A ratio & $1.58 \pm 1.33$ & $1.84 \pm 1.30$ & $1.40 \pm 1.35$ & 0.331 \\
\hline Septal e', cm/s & $5.37 \pm 2.15$ & $5.48 \pm 2.14$ & $5.30 \pm 2.21$ & 0.818 \\
\hline E/septal e' & $16.39 \pm 9.10$ & $15.31 \pm 5.32$ & $17.05 \pm 10.84$ & 0.609 \\
\hline
\end{tabular}

Abbreviations: ACEI, angiotensin-converting enzyme inhibitor; ADHF, acute decompensated heart failure; $\mathrm{ARB}$, angiotensin receptor blocker; BMI, body mass index; BNP, B-type natriuretic peptide; $\mathrm{CCB}$, calcium channel blocker; $\mathrm{Cr}$, creatinine; DM, diabetes mellitus; E/septal e', ratio of early mitral diastolic inflow velocity to medial mitral annular early diastolic velocity; EF, ejection fraction; HF, heart failure; Hs-CRP, high-sensitivity C-reactive protein; LDL-C, low-density lipoprotein cholesterol; MI, myocardial infarction; TC, total cholesterol. Data are presented as n (\%) or mean $\pm \mathrm{SD}$ unless otherwise specified. ${ }^{\text {a }} P$ value: systolic $\mathrm{HF}$ vs diastolic HF. 
F/U PWV from ADHF to CCHF

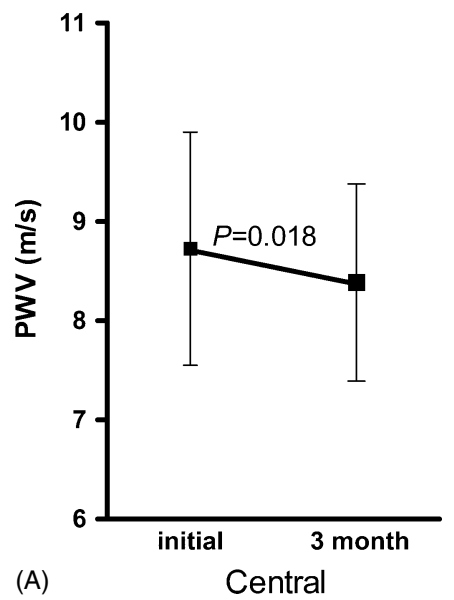

F/U PWW from ADHF to CCHF

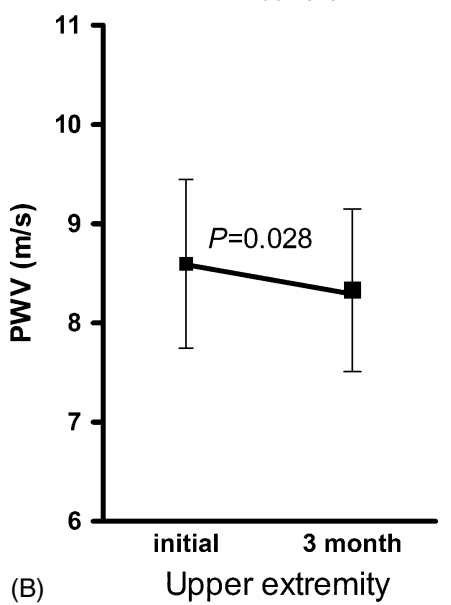

F/U PWV from ADHF to CCHF

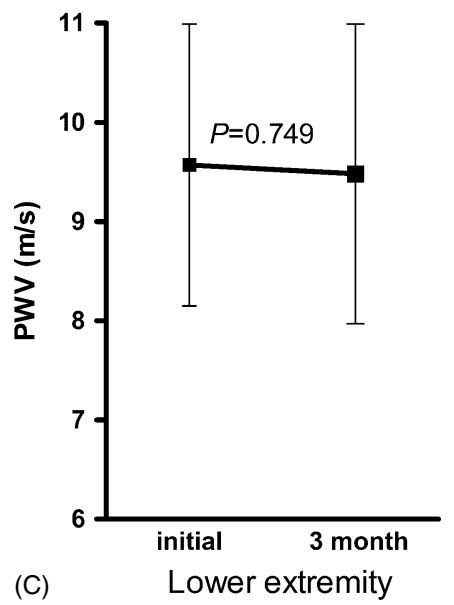

Figure 1. Improvement of PWV in $45 \mathrm{~F} / \mathrm{U}$ patients in (A) central, (B) upper-extremity, and (C) lower-extremity arteries. Abbreviations: ADHF, acute decompensated heart failure; CCHF, chronic compensated heart failure; F/U, follow-up; PWV, pulse wave velocity.

Table 2. Change of PWV in ADHF Patients Between Admission and 3 Months Follow-Up

\begin{tabular}{|c|c|c|c|c|}
\hline & PWV & Admission & $\begin{array}{l}\text { 3-Month } \\
\text { Follow-Up }\end{array}$ & $P$ Value \\
\hline \multicolumn{5}{|l|}{ LVEF } \\
\hline \multirow[t]{3}{*}{$\leq 40 \%, n=19$} & Central (m/s) & $8.53 \pm 1.13$ & $8.22 \pm 0.91$ & 0.203 \\
\hline & Upper (m/s) & $8.62 \pm 0.86$ & $8.49 \pm 0.91$ & 0.483 \\
\hline & Lower (m/s) & $9.77 \pm 1.34$ & $9.81 \pm 1.28$ & 0.937 \\
\hline \multirow[t]{3}{*}{$>40 \%, n=26$} & Central (m/s) & $8.87 \pm 1.20$ & $8.50 \pm 1.05$ & 0.044 \\
\hline & Upper (m/s) & $8.58 \pm 0.85$ & $8.21 \pm 0.74$ & 0.026 \\
\hline & Lower $(\mathrm{m} / \mathrm{s})$ & $9.42 \pm 1.48$ & $9.23 \pm 1.64$ & 0.604 \\
\hline \multicolumn{5}{|l|}{ HF etiology } \\
\hline \multirow[t]{3}{*}{ Ischemic, $n=31$} & Central (m/s) & $8.82 \pm 1.10$ & $8.44 \pm 0.95$ & 0.008 \\
\hline & Upper (m/s) & $8.59 \pm 0.87$ & $8.22 \pm 0.82$ & 0.011 \\
\hline & Lower $(\mathrm{m} / \mathrm{s})$ & $9.71 \pm 1.54$ & $9.73 \pm 1.64$ & 0.478 \\
\hline \multirow[t]{3}{*}{ Nonischemic, $n=14$} & Central (m/s) & $8.52 \pm 1.35$ & $8.27 \pm 1.12$ & 0.475 \\
\hline & Upper (m/s) & $8.61 \pm 0.82$ & $8.57 \pm 0.78$ & 0.720 \\
\hline & Lower $(\mathrm{m} / \mathrm{s})$ & $9.25 \pm 1.09$ & $8.92 \pm 1.00$ & 0.413 \\
\hline
\end{tabular}

Abbreviations: ADHF, acute decompensated heart failure; HF, heart failure; LVEF, left ventricular ejection fraction; PWV, pulse wave velocity. Data are presented as $n(\%)$ or mean \pm SD unless otherwise specified.

inefficient ventricular-arterial coupling is one. Ventriculararterial coupling is abnormal in patients with $\mathrm{HF} .{ }^{14}$ The coupling was quantified as $\mathrm{E}(\mathrm{es}) / \mathrm{E}(\mathrm{A})$, where $\mathrm{E}(\mathrm{es})$ is the slope of the end-systolic pressure-volume relation, and $\mathrm{E}(\mathrm{A})$ is an arterial elastance. In $\mathrm{ADHF}$, increased $\mathrm{E}(\mathrm{A})$, but not $\mathrm{E}(\mathrm{es})$, causes $\mathrm{E}(\mathrm{es}) / \mathrm{E}(\mathrm{A})$ to decrease. ${ }^{15,16}$ Inefficient ventricular-arterial coupling results in excessive myocardial energy expenditure for work performed. Existing data
Table 3. Hemodynamic Data and Medication Change From Admission to 3 Months in 45 Follow-Up Patients

\begin{tabular}{|c|c|c|c|}
\hline & Admission & $\begin{array}{l}\text { 3-Month } \\
\text { Follow-Up }\end{array}$ & $P$ Value \\
\hline \multicolumn{4}{|l|}{$\mathrm{BP}, \mathrm{mm} \mathrm{Hg}$} \\
\hline Peripheral systolic pressure & $118.1 \pm 17.1$ & $120.6 \pm 23.7$ & 0.467 \\
\hline Peripheral diastolic pressure & $72.0 \pm 11.8$ & $70.6 \pm 13.0$ & 0.565 \\
\hline Peripheral pulse pressure & $46.1 \pm 11.4$ & $50.0 \pm 16.1$ & 0.099 \\
\hline Heart rate, bpm & $72.5 \pm 11.2$ & $67.9 \pm 9.9$ & 0.061 \\
\hline$\beta$-Blocker & $26(58)$ & $24(53)$ & 0.832 \\
\hline $\mathrm{CCB}$ & $13(29)$ & $10(22)$ & 0.629 \\
\hline ACEI or ARB & $36(80)$ & $38(84)$ & 0.784 \\
\hline Digoxin & $25(56)$ & $27(60)$ & 0.831 \\
\hline Diuretic & $28(62)$ & $31(69)$ & 0.658 \\
\hline Lipid-lowering agent & $36(80)$ & $39(87)$ & 0.573 \\
\hline \multicolumn{4}{|c|}{$\begin{array}{l}\text { Abbreviations: ACEI, angiotensin-converting enzyme inhibitor; ARB, } \\
\text { angiotensin receptor blocker; BP, blood pressure; CCB, calcium channel } \\
\text { blocker. Data are presented as } n(\%) \text { or mean } \pm \text { SD unless otherwise } \\
\text { specified. }\end{array}$} \\
\hline
\end{tabular}

suggest that increased vascular loading may play a role in the development of ADHF. ${ }^{13}$ Our results showed higher central artery and upper-extremity artery stiffness in ADHF compared with CCHF. Therefore, our results suggest that increased central and upper-extremity artery stiffness leads to increased vascular loading, which is involved in the development of $\mathrm{ADHF}$, but the exact mechanisms underlying these increases remain unknown.

Although the factors causing arterial stiffness in $\mathrm{ADHF}$ remain to be determined, they may be speculated to include neurohormonal stimulation, endothelial dysfunction, and 
vascular wall edema. ${ }^{2}$ Previous studies have examined central and upper-extremity (but not lower-extremity) PWVs in CCHF (not ADHF) patients. ${ }^{17,18}$ However, they have reported conflicting findings. Mitchell et al. compared higher upper-extremity artery PWV in $28 \mathrm{CCHF}$ patients with relatively healthy controls, and found that both groups had similar central artery PWVs. ${ }^{17}$ Desai et al. reported that central artery PWV was higher in 16 preserved LVEF CCHF patients compared with healthy controls, whereas upperextremity artery PWVs were similar for both groups. ${ }^{18}$ Those 2 studies enrolled a small number of patients and differed in inclusion criteria, which may explain the conflicting findings.

We found improved central and upper-extremity PWVs at 3 months F/U. These results support a pathophysiological role for increased arterial stiffness in ADHF cases. This increased arterial stiffness may be due to vascular inflammation and edema caused by neurohormonal activation, a central feature of ADHF. ${ }^{19}$ However, clinical outcomes at 6 months $\mathrm{F} / \mathrm{U}$ showed no difference between the improvement and nonimprovement PWV groups. This might be caused by the small number of patients enrolled in our study.

There are a few studies regarding $\mathrm{HF}$ using lowerextremity PWV. Eliakim et al. reported no difference in lower-extremity PWV in patients with $\mathrm{CCHF}$ compared with age-matched healthy controls. ${ }^{20} \mathrm{We}$ found no change in lower-extremity PWV between ADHF and CCHF. We do not know the precise mechanisms for this, but there are a few possibilities that may explain the finding. Some HF patients had calcified lower-extremity arteries, but they were asymptomatic. ${ }^{21}$ Calcified arteries are often nondistensible, resulting in no change between ADHF and CCHF. Thus, we speculate that such occult lesions in the leg arteries may be one of the reasons for our findings. Further studies are needed to evaluate the mechanism of these findings.

We found that LDL-C may affect the change in PWV by multivariate logistic regression. More patients are needed to confirm this result. Table 2 showed that no change in PWV was shown in the systolic HF and nonischemic HF groups. We do not know the exact mechanism, but we assume that the difference of involved variables between the 2 groups led to this result.

In the present study, medication use was similar at admission and at the 3-month F/U. These findings suggest that PWV improved as a result of improved pathophysiology of the ADHF rather than medical therapy.

The present study has some limitations. First, the sample size was small. Second, 10 patients out of the 55 dropped out of the 3-month F/U. This might lead to a selection bias and impact our conclusions. Third, the study performed an initial echocardiographic examination but not a 3-month F/U echocardiographic examination; therefore, a more detailed analysis from $\mathrm{ADHF}$ to $\mathrm{CCHF}$ was not possible. Finally, hemoglobin and hematocrit levels were not recorded; therefore, it is possible that major changes in these parameters (or in blood viscosity) due to diuresis and resolution of HF were also contributory to the findings of altered PWV.

\section{Conclusion}

Central and upper-extremity PWVs improved as patients transitioned from $\mathrm{ADHF}$ to $\mathrm{CCHF}$. This suggests that increased artery stiffness has a relationship with the development of ADHF.

\section{References}

1. McMurray JJ, Stewart S. Epidemiology, aetiology, and prognosis of heart failure. Heart. 2000;83:596-602.

2. Et-Taouil K, Safar M, Plante GE. Mechanisms and consequences of large artery rigidity. Can J Physiol Pharmacol. 2003;81:205-211.

3. Hundley WG, Kitzman DW, Morgan TM, et al. Cardiac cycle-dependent changes in aortic area and distensibility are reduced in older patients with isolated diastolic heart failure and correlate with exercise intolerance. J Am Coll Cardiol. 2001;38:796-802.

4. Laskey WK, Kussmaul WG, Martin JL, et al. Characteristics of vascular hydraulic load in patients with heart failure. Circulation. 1985;72:61-71.

5. Zhang M, Bai Y, Ye $\mathrm{P}$, et al. Type 2 diabetes is associated with increased pulse wave velocity measured at different sites of the arterial system but not augmentation index in a Chinese population. Clin Cardiol. 2011;34:622-627.

6. Gaszner B, Lenkey Z, Illyes M, et al. Comparison of aortic and carotid arterial stiffness parameters in patients with verified coronary artery disease. Clin Cardiol. 2012;35:26-31.

7. Cruickshank K, Riste L, Anderson SG, et al. Aortic pulse-wave velocity and its relationship to mortality in diabetes and glucose intolerance: an integrated index of vascular function? Circulation. 2002;106:2085-2090.

8. Guerin AP, Blacher J, Pannier B, et al. Impact of aortic stiffness attenuation on survival of patients in end-stage renal failure. Circulation. 2001;103:987-992.

9. Laurent S, Boutouyrie P, Asmar R, et al. Aortic stiffness is an independent predictor of all-cause and cardiovascular mortality in hypertensive patients. Hypertension. 2001;37:1236-1241.

10. Shokawa T, Imazu M, Yamamoto $\mathrm{H}$, et al. Pulse wave velocity predicts cardiovascular mortality: findings from the Hawaii-Los Angeles-Hiroshima study. Circ J. 2005;69:259-264.

11. Sutton-Tyrrell K, Najjar SS, Boudreau RM, et al. Elevated aortic pulse wave velocity, a marker of arterial stiffness, predicts cardiovascular events in well-functioning older adults. Circulation. 2005; 111:3384-3390.

12. Wu AH, Packer M, Smith A, et al. Analytical and clinical evaluation of the Bayer ADVIA Centaur automated B-type natriuretic peptide assay in patients with heart failure: a multisite study. Clin Chem. 2004;50:867-873.

13. Cotter G, Felker GM, Adams KF, et al. The pathophysiology of acute heart failure-is it all about fluid accumulation? Am Heart J. 2008;155:9-18.

14. Asanoi H, Sasayama S, Kameyama T. Ventriculoarterial coupling in normal and failing heart in humans. Circ Res. 1989;65: 483-493.

15. Fox JM, Maurer MS. Ventriculovascular coupling in systolic and diastolic heart failure. Curr Heart Fail Rep. 2005;2:204-211.

16. Ohte N, Cheng CP, Little WC. Tachycardia exacerbates abnormal left ventricular-arterial coupling in heart failure. Heart Vessels. 2003;18:136-141.

17. Mitchell GF, Tardif JC, Arnold JM, et al. Pulsatile hemodynamics in congestive heart failure. Hypertension. 2001;38:1433-1439.

18. Desai AS, Mitchell GF, Fang JC, et al. Central aortic stiffness is increased in patients with heart failure and preserved ejection fraction. J Card Fail. 2009;15:658-664.

19. Ooi H, Chung W, Biolo A. Arterial stiffness and vascular load in heart failure. Congest Heart Fail. 2008;14:31-36.

20. Eliakim M, Sapoznikov D, Weinman J. Pulse wave velocity in healthy subjects and in patients with various disease states. $A m$ Heart J. 1971;82:448-457.

21. Hebert K, Lopez B, Michael C, et al. The prevalence of peripheral arterial disease in patients with heart failure by race and ethnicity. Congest Heart Fail. 2010;16:118-121. 